[3] W. S. Dorn, A symmetric dual theorem for quadratic programs, Journal of the Operations Research Society of Japan, 2 (1960) 93-97

[4] W. S. Dorn, Self-dual quadratic programs, Journal of the Society for Industrial and Applied Mathematics, 9 (1961) 51-54

[5] M. Frank and P. Wolfe, An algorithm for quadratic programming, Naval Research Logistics Quarterly, 3 (1956) 95-110

[6] D. Gale, The theory of linear economic models, McGraw-Hill, New York, 1960

[7] P. Wolfe, A duality theorem for nonlinear programming, Q. Appl. Math. 19 (1961) 239-244

\title{
APPLICATION OF VARIATIONAL PRINCIPLES TO LIMIT ANALYSIS*
}

BY T. MURA AND S. L. LEE (Civil Engineering Department, Northwestern University)

1. Introduction. The use of variational principles to derive kinematically admissible velocity fields and statically admissible stress fields in the limit analysis of perfectly plastic structures [1-6] has been discussed by several investigators [7-13]. The purpose of this paper is to demonstrate by means of these principles that the safety factor, the kinematically admissible multiplier and the statically admissible multiplier for a body made of perfectly plastic material and subjected to given surface traction are actually extremum values of the same functional under different constraint conditions.

The kinematically admissible multiplier $m^{*}$ is defined by the relation ${ }^{1}$

$$
m^{*}=k \int_{V}\left(2 \epsilon_{i}^{*} \epsilon_{i}^{*} \epsilon_{i}^{*}\right)^{1 / 2} d V / \int_{S_{T}} T_{i} v_{i}^{*} d S,
$$

where

$$
\begin{array}{rlrl}
\epsilon_{i j}^{*} & =\left(v_{i, j}^{*}+v_{i, i}^{*}\right) / 2 & \text { in } V, \\
\delta_{i j} v_{i, i}^{*} & =0 & & \text { in } V, \\
v_{i}^{*} & =0 & & \text { on } S_{V}, \\
\int_{S T} T_{i} v_{i}^{*} d S & >0 & &
\end{array}
$$

In these equations, $V$ denotes a closed domain bounded by a closed surface $S, S_{T}$ a portion of $S$ subjected to given surface traction $T_{i}$ while on the remainder $S_{V}$ the velocities are prescribed to vanish, $\epsilon_{i}^{*}$ the strain rate field associated with the velocity field $v_{i}^{*}$, and $k$ is the yield limit in simple shear. A velocity field is said to be kinematically admissible if it satisfies (3), (4) and (5).

The statically admissible multiplier is defined as follows: A stress field $\sigma_{i j}^{0}$ is said to be statically admissible if

$$
\begin{array}{ll}
\sigma_{i j, i}^{0}=0 & \text { in } V, \\
\sigma_{i j}^{0} n_{i}=m^{0} T_{i} & \text { on } S_{T},
\end{array}
$$

*Received October 4, 1962; revised manuscript received March 30, 1963. The research upon which this paper is based was supported in part by the Advanced Research Projects Agency of the Department of Defense, through Northwestern Materials Research Center.

'See [1], p. 247. 


$$
f\left(s_{i j}^{0}\right)=\frac{1}{2} s_{i}^{0} s_{i i}^{0}-k^{2} \leq 0 \text { in } V,
$$

where

$$
\begin{aligned}
s_{i i}^{0} & =\sigma_{i j}^{0}-\delta_{i j} \sigma^{0}, \\
\sigma^{0} & =1 / 3 \sigma_{k k}^{0} .
\end{aligned}
$$

The proportional constant $m^{0}$ defined by (7) is termed the statically admissible multiplier.

The ratio $s$ of a generic surface traction at the instant of impending plastic flow to the given value of the surface traction $T_{i}$ is called the safety factor. It is well known that

$$
m^{0} \leq s \leq m^{*}
$$

2. Safety factor. Consider the minimum problem of the functional $F_{1}\left[v_{i}, s_{i}\right]$ with independent arguments $v_{i}$ and $s_{i i}$. The existence of the minimum problem will be proven later.

Problem I. Minimize

$$
F_{1}\left[v_{i}, s_{i j}\right]=\int_{V} s_{i j} \frac{1}{2}\left(v_{i, j}+v_{i, i}\right) d V
$$

with the constraint conditions

$$
\begin{array}{r}
f\left(s_{i j}\right)=\frac{1}{2} s_{i j} s_{i j}-k^{2} \leq 0 \text { in } V, \\
\left(s_{i j}-s_{i j}^{\prime}\right) \frac{1}{2}\left(v_{i, i}+v_{i, i}\right) \geq 0 \text { in } V,
\end{array}
$$

for any $s_{i j}^{\prime}$ such that $f\left(s_{i j}^{\prime}\right) \leq 0$,

$$
\begin{aligned}
\delta_{i j} v_{i, j} & =0 \quad \text { in } \quad V, \\
v_{i} & =0 \text { on } S_{V}, \\
\int_{S T} T_{i} v_{i} d s & =1 .
\end{aligned}
$$

In order to determine the minimal conditions, the problem is transformed by employing point-function Lagrangian multipliers $\sigma, R_{i}$, and $\mu$ and a constant Lagrangian multiplier $m$ as follows.

Problem II. Extremize

$$
\begin{aligned}
F_{2}\left[v_{i}, s_{i j}, \sigma, R_{i}, m, \mu, \varphi\right] & =\int_{V} s_{i j} \frac{1}{2}\left(v_{i, i}+v_{i, i}\right) d V \\
+ & \int_{V} \sigma \delta_{i j} v_{i, i} d V-\int_{S_{V}} R_{i} v_{i} d S-m\left(\int_{S_{T}} T_{i} v_{i} d S-1\right) \\
& -\int_{V} \mu\left[f\left(s_{i j}\right)+\varphi^{2}\right] d V
\end{aligned}
$$

with the constraint condition

$$
\mu \geq 0 .
$$

The point function $\varphi$ is introduced on account of the inequality of condition (13) and, 
as will be shown later, constraint condition (19) is imposed in order to take into consideration constraint condition (14).

Taking the variation of $F_{2}$ leads to

$$
\begin{aligned}
\delta F_{2}=\int_{V} & \delta s_{i j} \frac{1}{2}\left(v_{i, i}+v_{i, i}\right) d V+\int_{V} s_{i j} \frac{1}{2}\left(\delta v_{i, j}+\delta v_{i, i}\right) d V \\
& +\int_{V} \delta \sigma \delta_{i j} v_{i, j} d V+\int_{V} \sigma \delta_{i j} \delta v_{i, j} d V-\int_{S_{V}} \delta R_{i} v_{i} d S-\int_{S V} R_{i} \delta v_{i} d S \\
& -\delta m\left(\int_{S T} T_{i} v_{i} d S-1\right)-m \int_{S T} T_{i} \delta v_{i} d S-\int_{V} \delta \mu\left[f\left(s_{i j}\right)+\varphi^{2}\right] d V \\
& -\int_{V} \mu \frac{\partial f}{\partial s_{i i}} \delta s_{i j} d V-\int_{V} \mu 2 \varphi \delta \varphi d V
\end{aligned}
$$

Integrating (20) by parts yields the natural conditions

$$
\begin{aligned}
\frac{1}{2}\left(v_{i, i}+v_{i, i}\right) & =\mu \frac{\partial f}{\partial s_{i j}} \quad \text { in } \quad V, \\
\mu & \geq 0, \\
\left(s_{i i}+\delta_{i j} \sigma\right)_{, i} & =0 \quad \text { in } \quad V, \\
\left(s_{i j}+\delta_{i j} \sigma\right) n_{i} & =m T_{i} \quad \text { on } \quad S_{T}, \\
\left(s_{i j}+\delta_{i j} \sigma\right) n_{j} & =R_{i} \quad \text { on } \quad S_{V}, \\
f\left(s_{i i}\right)+\varphi^{2} & =0 \quad \text { in } \quad V, \\
\mu \varphi & =0 \quad \text { in } \quad V, \\
\delta_{i j} v_{i, j} & =0 \quad \text { in } \quad V, \\
v_{i} & =0 \quad \text { on } \quad S_{V}, \\
\int_{S T} T_{i} v_{i} & =1 .
\end{aligned}
$$

Condition (21) is the plastic potential flow law, (22) to (24) are the equilibrium conditions, and (27) to (29) define a kinematically admissible velocity field. Conditions (25) and (26) define the admissible domain of the stress space, i.e.,

$$
\begin{aligned}
& f\left(s_{i i}\right)=0 \quad \text { if } \quad \mu>0, \\
& f\left(s_{i i}\right) \leq 0 \quad \text { if } \quad \mu=0 .
\end{aligned}
$$

Obviously, (21) to (29) are the conditions for incipient plastic flow. Condition (24) should be understood as an equation for determining $R_{i}$, the reaction on the boundary, which is arbitrary. It should be mentioned here that condition (29) is no more restrictive than the requirement

$$
\int_{S T} T_{i} v_{i} d S>0 .
$$

Setting the integral equal to unity only determines the scale of the otherwise arbitrary size of the velocity vector. It should also be observed that constraint condition (14) on 
$F_{1}$ is satisfied by $(21)$ and the fact that the yield surface, $f\left(s_{i j}\right)=0$, is convex with respect to the origin of the vector space.

Integrating the functionals by parts in view of (21) to (29), it can be readily shown that the minimum value of $F_{1}$ and the extremum value of $F_{2}$ are equal to $m$. Therefore the safety factor can be defined as the minimum of $F_{1}$ or the extremum value of $F_{2}$, i.e.,

$$
\text { Min. } F_{1}=\text { Ext. } F_{2}=m=s
$$

To prove the existence of the minimum problem for $F_{1}$, it is sufficient to show that

$$
\int_{V} s_{i, \frac{1}{2}}^{*}\left(v_{i, j}^{*}+v_{i, i}^{*}\right) d V \geq \int_{V} s_{i j} \frac{1}{2}\left(v_{i, i}+v_{i, i}\right) d V
$$

for an set of $v_{i}^{*}$ and $s_{i j}^{*}$ which satisfy the constraint conditions (13) to (17) and $v_{i}$ and $s_{i j}$ are the stationary functions. Since conditions (22) to (29) are applicable to $v_{i}$ and $s_{i j}$,

$$
\begin{aligned}
\int_{V} s_{i j} \frac{1}{2}\left(v_{i, j}+v_{i, i}\right) d V & =\int_{V} \sigma_{i j} v_{i, i} d V=\int_{S T} \sigma_{i j} v_{i} n_{j} d S \\
& =m \int_{S T} T_{i} v_{i} d S=m \int_{S T} T_{i} v_{i}^{*} d S=\int_{S} \sigma_{i j} v_{i}^{*} n_{i} d S \\
& =\int_{V} \sigma_{i j, j} v_{i}^{*} d V+\int_{V} \sigma_{i j} v_{i, j}^{*} d V=\int_{V} s_{i j} \frac{1}{2}\left(v_{i, j}^{*}+v_{i, i}^{*}\right) d V
\end{aligned}
$$

In view of (14),

$$
\left(s_{i j}^{*}-s_{i j}\right) \frac{1}{2}\left(v_{i, i}^{*}+v_{i, i}^{*}\right) \geq 0
$$

Thus, statement (33) is established and the existence of the minimum problem is verified.

3. Kinematically admissible multiplier. It can be shown that a kinematically admissible velocity field $v_{i}^{*}$ and the associated stress field $s_{i j}^{*}$ constructed from $v_{i}^{*}$ by means of

$$
s_{i j}^{*}=k \epsilon_{i i}^{*} /\left(\frac{1}{2} \epsilon_{m n}^{*} \epsilon_{m n}^{*}\right)^{1 / 2},
$$

where $\epsilon_{i j}^{*}$ is defined by (2), are admissible comparison functions of Problem I. As a matter of fact, substituting

$$
\begin{gathered}
v_{i}=v_{i}^{*} / \int_{S T} T_{i} v_{i}^{*} d S, \\
s_{i j}=s_{i j}^{*}
\end{gathered}
$$

in (12) yields

$$
F_{1}=m^{*},
$$

where $m^{*}$ is as defined by (1). Since the safety factor is the minimum value of $F_{1}$, the following inequality holds:

$$
m^{*} \geq s,
$$

4. Statically admissible multiplier. In accordance with the maximal minimum principle of calculus of variations ${ }^{2}$ another minimum problem may be obtained from

'See [14], p. 232. 
Problem II for fixed values of the Lagrangian multipliers $\sigma^{0}, R_{i}^{0}, m^{0}$ and $\mu^{0}$ with additional constraint conditions as follows.

Problem III. Minimize

$$
\begin{aligned}
F_{3}\left[v_{i}, s_{i j}\right]=\int_{V} & s_{i j} \frac{1}{2}\left(v_{i, j}+v_{i, i}\right) d V+\int_{V} \sigma^{0} \delta_{i j} v_{i, j} d V \\
& \quad-\int_{S V} R_{i}^{0} v_{i} d S-m^{0}\left(\int_{S_{T}} T_{i} v_{i} d S-1\right)-\int_{V} \mu^{0}\left[f\left(s_{i j}\right)+\varphi^{2}\right] d V
\end{aligned}
$$

with constraint conditions

$$
\begin{array}{r}
f\left(s_{i j}\right)+\varphi^{2}=0 \text { in } V, \\
\left(s_{i j}-s_{i j}^{\prime}\right) \frac{1}{2}\left(v_{i, j}+v_{i, i}\right) \geq 0 \text { in } V,
\end{array}
$$

for any $s_{i j}^{\prime}$ such that $f\left(s_{i j}^{\prime}\right) \leq 0$.

Taking the variation of $F_{3}$ with respect to $v_{i}$ leads to

$$
\begin{aligned}
& \left(s_{i j}+\delta_{i j} \sigma^{0}\right)_{, i}=0 \quad \text { in } V \text {, } \\
& \left(s_{i j}+\delta_{i j} \sigma^{0}\right) n_{i}=m^{0} T_{i} \text { on } S_{T} \text {, } \\
& \left(s_{i j}+\delta_{i j} \sigma^{0}\right) n_{j}=R_{i}^{0} \quad \text { on } \quad S_{V} \text {. }
\end{aligned}
$$

Integrating (41) by parts in view of (44) to (46) leads to

$$
F_{3}=m^{0}-\int_{V} \mu^{0}\left[f\left(s_{i j}\right)+\varphi^{2}\right] d V
$$

which, in view of (42), simplifies finally into

$$
\text { Min. } F_{3}=m^{0} \text {. }
$$

In order to prove that Problem III is a minimum problem, it is sufficient to show that for any set of arbitrary comparison functions $v_{i}^{0}$ and $s_{i j}^{0}$,

$$
F_{3}\left[v_{i}^{0}, s_{i i}^{0}\right] \geq F_{3}\left[v_{i}, s_{i j}\right]
$$

in which $v_{i}$ and $s_{i j}$ are the stationary functions. In view of (47),

$$
F_{3}\left[v_{i}, s_{i j}\right]=F_{3}\left[v_{i}^{0}, s_{i j}\right]
$$

Therefore, taking (42) and (43) into consideration,

$$
\begin{aligned}
& F_{3}\left[v_{i}^{0}, s_{i j}^{0}\right]-F_{3}\left[v_{i}, s_{i j}\right]=F_{3}\left[v_{i}^{0}, s_{i j}^{0}\right]-F_{3}\left[v_{i}^{0}, s_{i j}\right] \\
& \quad=\int_{V}\left(s_{i j}^{0}-s_{i j}\right) \frac{1}{2}\left(v_{i, i}^{0}+v_{i, i}^{0}\right) d V \geq 0
\end{aligned}
$$

and (49) holds true.

Problem I may be made up from Problem III by superimposing, on top of (42) and (43), the following additional constraint conditions:

$$
\begin{aligned}
\delta_{i j} v_{i, j} & =0 \quad \text { in } \quad V, \\
v_{i} & =0 \quad \text { on } \quad S_{V}, \\
\int_{S T} T_{i} v_{i} d S & =1 .
\end{aligned}
$$


Since (52) to (54) are not natural conditions of Problem III, they raise the minimum value of the functional. Hence the minimum of $F_{1}$ cannot be less than the minimum value of $F_{3}$. In other words,

$$
m^{0} \leq s
$$

Since conditions (42), (44) and (45) are identical to (6), (7) and (8), $m^{0}$ in (55) turns out to be a statically admissible multiplier.

5. Conclusion. From the foregoing discussions, the statically admissible multiplier $m^{0}$ can be defined as the minimum value of $F_{3}$ with the constraint conditions (42) and (43), or the value of $F_{3}$ calculated from a statically admissible stress field.

Since a kinematically admissible velocity field satisfies conditions (52) to (54) and $F_{3}=F_{1}$ under these additional constraint conditions, the kinematically admissible multiplier $m^{*}$ can be defined as the value of $F_{3}$ or $F_{1}$ calculated from a kinematically admissible velocity field and a stress field with constraint conditions (42) and (43).

The safety factor can be defined as the maximal minimum value of $F_{3}$ with respect to the Lagrangian multipliers $\sigma^{0}, R_{i}^{0}, m^{0}$ and $\mu^{0}$, or the minimum value of $F_{3}$ or $F_{1}$ with the constraint conditions (42), (43), (52), (53) and (54). It can also be defined as the extremum value of $F_{2}$ with the constraint condition (19).

It should be noted that the foregoing discussions can be extended to the analysis of anisotropic solids by using the proper $f\left(s_{i j}\right)$. Cases involving discontinuous velocity fields can be treated by considering the additional work done on the surface of discontinuity.

\section{REFERENCES}

1. W. Prager and P. G. Hodge, Theory of perfectly plastic solids, John Wiley \& Sons, New York (1951)

2. P. S. Symonds and W. Prager, J. Appl. Mech. 17, 315 (1950)

3. P. S. Symonds, J. Appl. Mech. 18, 85 (1951)

4. D. C. Drucker, H. J. Greenberg and W. Prager, J. Appl. Mech. 18, 371 (1951)

5. P. S. Symonds and B. G. Neal, J. Franklin Inst. 252, 383 and 469 (1951)

6. D. C. Drucker, W. Prager and H. J. Greenberg, Q. Appl. Math. 9, 381 (1952)

7. M. A. Sadowsky, J. Appl. Mech. 10, A65 (1943)

8. W. Prager, J. Appl. Mech. 10, A65 (1943)

9. G. H. Handelman, Q. Appl. Math. 1, 351 (1944)

10. A. A. Markov, Prik. Mat. Mekh. 11, 339 (1947)

11. R. Hill, Q. J. Mech. Appl. Math. 1, 18 (1948)

12. H. J. Greenberg, Q. Appl. Math. 7, 85 (1949)

13. D. C. Drucker, Proc. Symp. Appl. Math. 8, 7 (1958)

14. R. Courant and D. Hilbert, Methods of mathematical Physics, Vol. I, Interscience Publications, New York (1953) 